\title{
Oncologic and long-term outcomes of enhanced recovery after surgery in cancer surgeries - a systematic review
}

\author{
Qianyun Pang, Liping Duan, Yan Jiang and Hongliang Liu* (D)
}

\begin{abstract}
Background: Clinical evidence has proved that enhanced recovery after surgery (ERAS) can improve short-term clinical outcomes after various types of surgeries, but the long-term benefits have not yet been examined, especially with respect to cancer surgeries. Therefore, a systematic review of the current evidence was conducted.

Methods: The Pubmed, Cochrane Library, Embase, and Web of Science databases were searched using the following key words as search terms: "ERAS" or "enhanced recovery" or "fast track", "oncologic outcome", "recurrence", "metastasis", "long-term outcomes", "survival", and "cancer surgery". The articles were screened using the inclusion and exclusion criteria, and the data from the included studies were extracted and analyzed.

Results: A total of twenty-six articles were included in this review. Eighteen articles compared ERAS and conventional care, of which, 12 studies reported long-term overall survival (OS), and only 4 found the improvement by ERAS. Four studies reported disease-free survival (DFS), and only 1 found the improvement by ERAS. Five studies reported the outcomes of return to intended oncologic treatment after surgery (RIOT), and 4 found improvements in the ERAS group. Seven studies compared high adherence to ERAS with low adherence, of which, 6 reported the long-term OS, and 3 showed improvements by high adherence. One study reported high adherence could reduce the interval from surgery to RIOT. Four studies reported the effect of altering one single item within the ERAS protocol, but the results of 2 studies were controversial regarding the long-term OS between laparoscopic and open surgery, and 1 study showed improvements in OS with restrictive fluid therapy.

Conclusions: The use of ERAS in cancer surgeries can improve the on-time initiation and completion of adjuvant chemotherapy after surgery, and the high adherence to ERAS can lead to better outcomes than low adherence. Based on the current evidence, it is difficult to determine whether the ERAS protocol is associated with long-term overall survival or cancer-specific survival.
\end{abstract}

Keywords: Enhanced recovery after surgery, Cancer surgery, Long-term outcome, Oncologic outcome

\section{Background}

The concept of enhanced recovery after surgery (ERAS) regimen was first introduced by Kehlet in 1997 [1]; it was then gradually accepted and widely used in nearly all types of surgeries. Clinical evidence has proved that

\footnotetext{
* Correspondence: liuhl75@163.com

Department of Anesthesiology, Chongqing University Cancer Hospital, Hanyu Road No. 181, Shapingba District, Chongqing 400030, China
}

ERAS not only improves clinical outcomes and quality of care, but also significantly reduces the cost of hospitalization [2-6]. However, the majority of the clinical evidence regarding the benefits of ERAS describes short-term outcomes; the long-term benefits of ERAS are not fully elucidated, especially with respect to cancer surgeries.

\section{$\triangle B M C$}

(c) The Author(s). 2021 Open Access This article is licensed under a Creative Commons Attribution 4.0 International License, which permits use, sharing, adaptation, distribution and reproduction in any medium or format, as long as you give appropriate credit to the original author(s) and the source, provide a link to the Creative Commons licence, and indicate if changes were made. The images or other third party material in this article are included in the article's Creative Commons licence, unless indicated otherwise in a credit line to the material. If material is not included in the article's Creative Commons licence and your intended use is not permitted by statutory regulation or exceeds the permitted use, you will need to obtain permission directly from the copyright holder. To view a copy of this licence, visit http://creativecommons.org/licenses/by/4.0/ The Creative Commons Public Domain Dedication waiver (http://creativecommons.org/publicdomain/zero/1.0/) applies to the data made available in this article, unless otherwise stated in a credit line to the data. 
Due to the increasing number of cancer cases, the proportion of cancer surgeries among all surgeries is increasing worldwide. Patients undergoing cancer surgeries commonly need neoadjuvant chemotherapy afterwards, and the on-time initiation and completion of chemotherapy after surgery are critical for the prognosis [7], as recurrence and metastasis can directly influence quality of life and long-term survival. The short-term benefits of ERAS are postulated to be associated with its long-term benefits [8], but this has not been fully verified for cancer surgeries. Thus, we conducted a literature search to identify studies on oncologic and long-term outcomes that examined: (1) ERAS versus conventional care, (2) high adherence to ERAS versus low adherence, and (3) the effects of altering one single item within the ERAS protocol.

\section{Methods}

We construct the framework of this systematic review in accordance with the 2009 PRISMA (Preferred Reporting Items for Systematic Reviews and Meta-Analyses) guidelines [9]. The PRISMA checklist is presented in Additional file 1 . The protocol of this systematic review was registered in INPLASY with the registration number INPLASY202150099, and the link https://inplasy.com/ inplasy-2021-5-0099/.

\section{Literature search}

The Pubmed, Cochrane Library, Embase, and Web of Science databases were searched from Jan 2000 to April 2021. The key terms were "ERAS" or "enhanced recovery" or "fast track", "oncologic outcome", "recurrence", "metastasis", "long-term outcomes", "survival", "cancer surgery", and various combinations of these key terms were used. The detailed search strategy in Pubmed was shown as followed:

\#1 "Enhanced Recovery After Surgery"[Mesh]

\#2 ((Enhanced Recovery After Surgery) OR (ERAS))

OR (fast track)

\#3 Cancer Surgery

\#4. (\#1 OR \#2) AND \#3

\#5 ((((( oncologic outcome) OR (recurrence)) OR (metastasis)) OR (long-term outcomes)) OR (survival)) OR (cancer specific death)) OR (oncologic)

\#6 \#4. AND \#5

The detailed search strategies in other databases were presented in Additional file 2. The reference lists of the included studies were checked for potentially eligible articles. The languages of the full-text articles were restricted to English and Chinese.

\section{Inclusion and exclusion criteria}

The inclusion criteria included studies comparing ERAS and conventional care, comparing different levels of adherence to ERAS, examining alterations of one single item within the ERAS protocol, studies with adult patients ( $>18$ years old) undergoing cancer surgery, studies describing oncologic outcomes (return to intended oncologic treatment after surgery (RIOT), recurrence, metastasis, and cancer-specific survival) or long-term outcomes (overall survival and quality of life), and prospective or retrospective studies. Exclusion criteria included studies of pediatric surgery, studies describing only short-term outcomes, studies without full text, review articles, or case reports.

\section{Data extraction}

Two reviewers (QP and LD) independently extracted data from all included articles, which included the author, publication time, study design, patient age, major diagnosis, surgical type, patient groups and sample size, key elements of the ERAS protocol, oncologic and longterm outcomes, and findings. Disagreements were resolved through consensus between reviewers; if necessary, an additional reviewer was consulted to resolve the dispute.

\section{Data analysis}

The methodological quality was evaluated by the Newcastle-Ottawa Scale (NOS) for cohort studies and by the Jadad score for RCTs. The highest NOS score was of 9 stars and the highest Jadad score was 7. Information on major outcomes of interest was recorded, including oncologic outcomes (RIOT, recurrence, metastasis, and cancer-specific survival), long-term overall survival, and quality of life.

\section{Results}

\section{Literature search and retrieval}

A total of 845 relevant publications were identified through the keywords. The full versions of 35 articles were retrieved after screening and conducting a detailed selection process, and 26 articles [10-35] eventually met the inclusion criteria and underwent data extraction. The details of the screening process are presented in Fig. 1.

\section{Study characteristics}

The characteristics and quality of the included articles are presented in Table 1 . Of these 26 articles, 3 were randomized trials [12, 13, 15], which were evaluated by Jadad score, and the other 23 were prospective or retrospective cohort studies, which were evaluated by the NOS. Eighteen articles compared conventional care with ERAS [1021, 29-34], 7 studies compared high adherence to ERAS with low adherence [16, 22-26, 34], and 4 studies [27-29, 35] investigated the outcome of one single item altered within ERAS protocol. The elements of the ERAS protocol in each article are presented in Table 2. The number of 


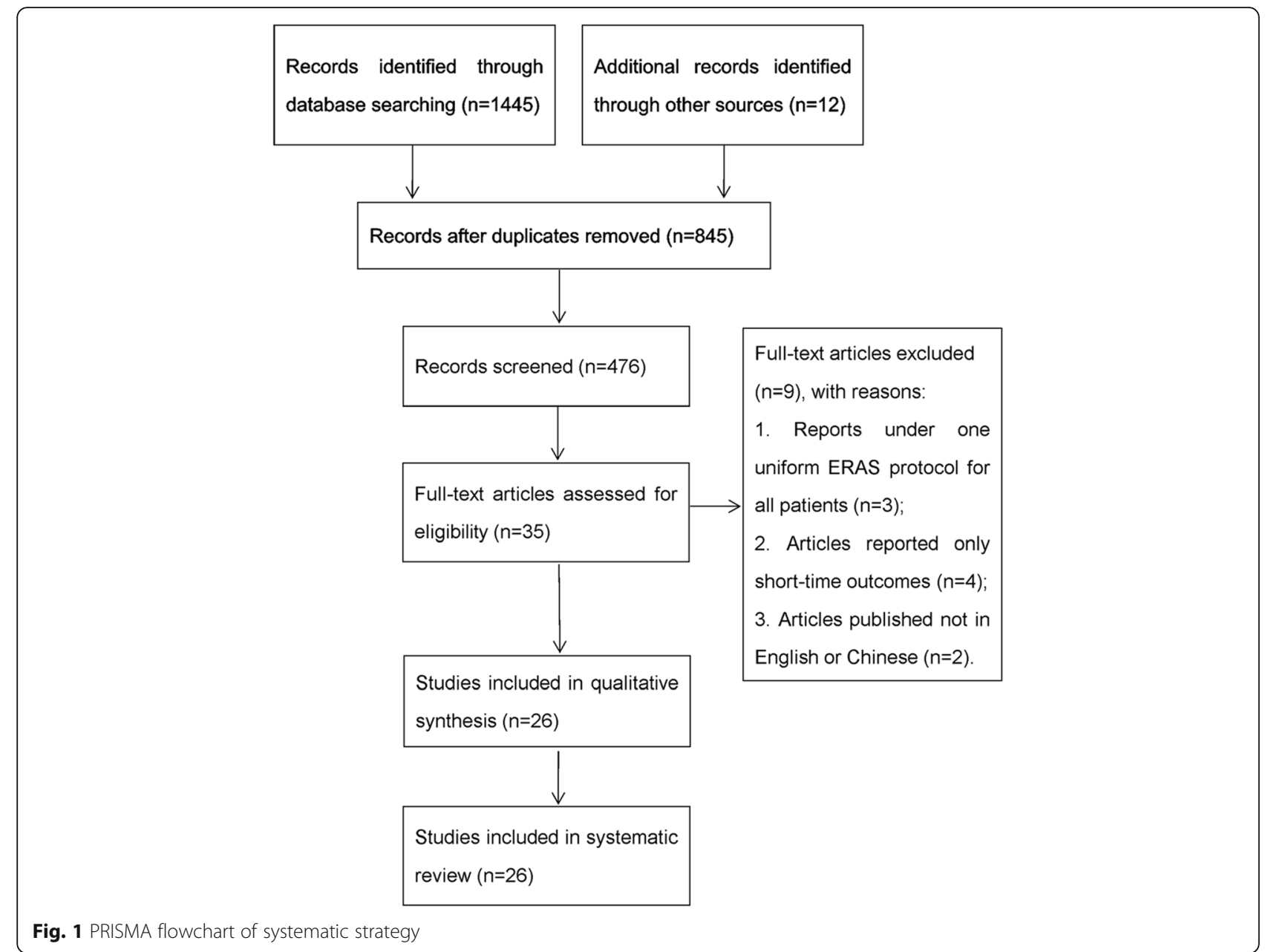

ERAS elements in the included articles ranged from 3 to 18 , and 17 articles described a protocol containing more than 10 elements $[11,13-18,21,22,24,26,27,29,30$, 32-34].

\section{Outcomes and findings}

The outcomes and findings of the included studies are displayed in Table 3. Seventeen studies described oncologic outcomes [11, 12, 16-23, 25, 28-31, 33, 35], and 20 studies described long-term outcomes [10-16, 22-34].

\section{Non-ERAS vs ERAS}

Twelve studies [10-14, 16, 29-34] reported long-term overall survival (OS), and the follow-up time ranged from 10 months to 10 years. Four studies [14, 31, 33, 34] showed ERAS was associated with increasing long-term OS, while the others found no differences between conventional care and ERAS. One study [15] reported quality of life, and the results showed that ERAS improved 6-month quality of life after surgery.

Three studies reported long-term cancer-specific survival (CSS) [11, 12, 33], and 4 studies reported long- term disease-free survival (DFS) [16, 29-31].The followup time ranged from 10 months to 10 years, 1 study showed that ERAS could improve CSS [33] and DFS [30], while the others found no differences. One study [31] reported recurrence and metastasis, the result found no difference between conventional care and ERAS.

Five studies [17-21] reported the outcomes of RIOT; 2 out of the 5 studies showed a reduced interval from surgery to RIOT with the ERAS group [18, 21], 1 showed improvement in RIOT completion with ERAS [20], 1 showed an improvement in the rate of on time to RIOT with ERAS [19], and 1 showed no differences in the completion of RIOT and the interval from surgery to RIOT between non-ERAS and ERAS groups [17].

\section{High adherence to ERAS vs low adherence to ERAS}

Seven studies compared high adherence to ERAS and low adherence to ERAS [16, 22-26, 34]. Long-term OS was reported in six studies, and the follow-up time ranged from 2 to 5 years. Half of the studies showed no differences in OS between high and low adherence [16, 22, 26], and half showed improvements in OS by high 
Table 1 Characteristics of included studies

\begin{tabular}{|c|c|c|c|c|c|c|c|}
\hline Author & Year & Study design & Surgery & Groups & $\begin{array}{l}\text { Patient } \\
\text { age (year) }\end{array}$ & Outcomes & $\begin{array}{l}\text { NOS/ } \\
\text { Jadad } \\
\text { score* }^{*}\end{array}$ \\
\hline \multicolumn{8}{|c|}{ Non-ERAS vs ERAS } \\
\hline $\begin{array}{l}\text { Oakley et al. } \\
{[10]}\end{array}$ & 2016 & $\begin{array}{l}\text { Retrospective } \\
\text { cohort }\end{array}$ & $\begin{array}{l}\text { Esophagi-gastric } \\
\text { resection }\end{array}$ & $\begin{array}{l}\text { Non-ERAS }(n=81) \\
\text { ERAS }(n=66)\end{array}$ & $\begin{array}{l}\text { Median } \\
78.8 \\
\text { Median } \\
78.5\end{array}$ & OS & 8 \\
\hline $\begin{array}{l}\text { Pang et al. } \\
\text { [11] }\end{array}$ & 2018 & $\begin{array}{l}\text { Prospective } \\
\text { cohort }\end{array}$ & Radical cystectomy & $\begin{array}{l}\text { Non-ERAS }(n=393) \\
\text { ERAS }(n=60)\end{array}$ & $\begin{array}{l}\text { Median } 66 \\
\text { Median } 71\end{array}$ & $\begin{array}{l}\text { OS } \\
\text { CSS }\end{array}$ & 7 \\
\hline $\begin{array}{l}\text { Ziegelmueller } \\
\text { et al. [12] }\end{array}$ & 2019 & $\begin{array}{l}\text { Prospective } \\
\text { randomized }\end{array}$ & Radical cystectomy & $\begin{array}{l}\text { Non-ERAS }(n=38) \\
\text { ERAS }(n=60)\end{array}$ & $\begin{array}{l}\text { Median } \\
67.5 \\
\text { Median } 70\end{array}$ & $\begin{array}{l}\text { OS } \\
\text { CSS }\end{array}$ & $4^{*}$ \\
\hline Ye [13] & 2017 & $\begin{array}{l}\text { Prospective } \\
\text { randomized }\end{array}$ & Radical gastrectomy & $\begin{array}{l}\text { Non-ERAS }(n=38) \\
\text { ERAS }(n=47)\end{array}$ & $\begin{array}{l}\text { Mean } 43.2 \\
\text { Mean } 45.2\end{array}$ & OS & $4^{*}$ \\
\hline $\begin{array}{l}\text { Yang et al. } \\
{[14]}\end{array}$ & 2020 & $\begin{array}{l}\text { Retrospective } \\
\text { cohort }\end{array}$ & Radical gastrectomy & $\begin{array}{l}\text { Non-ERAS }(n=521) \\
\text { ERAS }(n=521)\end{array}$ & Mean 63 & OS & 8 \\
\hline Liu et al. [15] & 2020 & $\begin{array}{l}\text { Randomized } \\
\text { control trial }\end{array}$ & Craniotomy & $\begin{array}{l}\text { Non-ERAS }(n=29) \\
\text { ERAS }(n=36)\end{array}$ & $18-65$ & Life quality & $6^{*}$ \\
\hline $\begin{array}{l}\text { Passeri et al. } \\
{[16]}\end{array}$ & 2020 & $\begin{array}{l}\text { Prospective } \\
\text { cohort }\end{array}$ & $\begin{array}{l}\text { Pancreatico- } \\
\text { duodenectomy }\end{array}$ & $\begin{array}{l}\text { Non-ERAS }(n=86) \\
\text { ERAS }(n=86)\end{array}$ & Median 67 & $\begin{array}{l}\text { OS } \\
\text { DFS }\end{array}$ & 6 \\
\hline Day et al. [17] & 2015 & $\begin{array}{l}\text { Prospective } \\
\text { cohort }\end{array}$ & Hepatectomy & $\begin{array}{l}\text { Non-ERAS }(n=43) \\
\text { ERAS }(n=75)\end{array}$ & $\begin{array}{l}\text { Median } 60 \\
\text { Median } 59\end{array}$ & $\begin{array}{l}\text { Failure to RIOT } \\
\text { Days to RIOT }\end{array}$ & 8 \\
\hline $\begin{array}{l}\text { Lohsiriwat } \\
{[18]}\end{array}$ & 2014 & $\begin{array}{l}\text { Prospective } \\
\text { cohort }\end{array}$ & $\begin{array}{l}\text { Colorectal cancer } \\
\text { resection }\end{array}$ & $\begin{array}{l}\text { Non-ERAS }(n=40) \\
\text { ERAS }(n=20)\end{array}$ & $\begin{array}{l}\text { Mean } 62 \\
\text { Mean } 57.6\end{array}$ & Days to RIOT & 7 \\
\hline $\begin{array}{l}\text { Hassinger } \\
\text { et al. [19] }\end{array}$ & 2019 & $\begin{array}{l}\text { Retrospective } \\
\text { cohort }\end{array}$ & $\begin{array}{l}\text { Colorectal cancer } \\
\text { resection }\end{array}$ & $\begin{array}{l}\text { Non-ERAS }(n=174) \\
\text { ERAS }(n=189)\end{array}$ & $\begin{array}{l}\text { Median } \\
61.1 \\
\text { Median } \\
62.4\end{array}$ & Rate of on time to RIOT & 8 \\
\hline $\begin{array}{l}\text { Nelson et al. } \\
{[20]}\end{array}$ & 2019 & $\begin{array}{l}\text { Prospective } \\
\text { cohort }\end{array}$ & Lung resection & $\begin{array}{l}\text { Non-ERAS }(n=230) \\
\text { ERAS }(n=92)\end{array}$ & Median 66 & $\begin{array}{l}\text { Days to RIOT } \\
\text { Rate of completing RIOT }\end{array}$ & 7 \\
\hline Li et al. [21] & 2020 & $\begin{array}{l}\text { Prospective } \\
\text { cohort }\end{array}$ & $\begin{array}{l}\text { Pancreatico- } \\
\text { duodenectomy }\end{array}$ & $\begin{array}{l}\text { Non-ERAS }(n=141) \\
\text { ERAS }(n=203)\end{array}$ & $\begin{array}{l}\text { Median } \\
58.2 \\
\text { Median } \\
58.9\end{array}$ & Days to RIOT & 7 \\
\hline $\begin{array}{l}\text { Wang et al. } \\
{[29]}\end{array}$ & 2021 & $\begin{array}{l}\text { Retrospective } \\
\text { cohort }\end{array}$ & $\begin{array}{l}\text { Colorectal cancer } \\
\text { resection }\end{array}$ & $\begin{array}{l}\text { Non-ERAS }(1)(n= \\
142) \\
\text { ERAS }(1)(n=125) \\
\text { Non-ERAS }(2)(n= \\
138) \\
\text { ERAS (2) }(n=137)\end{array}$ & $\begin{array}{l}\text { Median } \\
57.65 \\
\text { Median } \\
56.22 \\
\text { Median } \\
58.45 \\
\text { Median } \\
57.18\end{array}$ & OS; DFS & 7 \\
\hline $\begin{array}{l}\text { Zhang et al. } \\
\text { [30] }\end{array}$ & 2021 & $\begin{array}{l}\text { Retrospective } \\
\text { cohort }\end{array}$ & Hepatectomy & $\begin{array}{l}\text { Non-ERAS }(n=463) \\
\text { ERAS }(n=463)\end{array}$ & $\begin{array}{l}\text { Median } \\
62.8 \\
\text { Median } \\
63.2\end{array}$ & OS; DFS & 7 \\
\hline $\begin{array}{l}\text { Quiram et al. } \\
\text { [31] }\end{array}$ & 2019 & $\begin{array}{l}\text { Retrospective } \\
\text { cohort }\end{array}$ & Rectal cancer resection & $\begin{array}{l}\text { Non-ERAS }(n=280) \\
\text { ERAS }(n=320)\end{array}$ & $\begin{array}{l}\text { Median } \\
58.9 \\
\text { Median } \\
58.2\end{array}$ & $\begin{array}{l}\text { OS; DFS; local recurrence; } \\
\text { metastasis }\end{array}$ & 7 \\
\hline Zhu et al. [32] & 2019 & $\begin{array}{l}\text { Retrospective } \\
\text { cohort }\end{array}$ & $\begin{array}{l}\text { Pancreatico- } \\
\text { duodenectomy }\end{array}$ & $\begin{array}{l}\text { Non-ERAS }(n=69) \\
\text { ERAS }(n=64)\end{array}$ & $\begin{array}{l}\text { Median } \\
64.1 \\
\text { Median } \\
64.3\end{array}$ & OS & 6 \\
\hline Tian et al. [33] & 2020 & $\begin{array}{l}\text { Retrospective } \\
\text { cohort }\end{array}$ & Gastrectomy & $\begin{array}{l}\text { Non-ERAS }(n=365) \\
\text { ERAS }(n=365)\end{array}$ & $\begin{array}{l}\text { Median } \\
59.4 \\
\text { Median } \\
59.5\end{array}$ & OS; CSS & 7 \\
\hline
\end{tabular}


Table 1 Characteristics of included studies (Continued)

\begin{tabular}{|c|c|c|c|c|c|c|c|}
\hline Author & Year & Study design & Surgery & Groups & $\begin{array}{l}\text { Patient } \\
\text { age (year) }\end{array}$ & Outcomes & $\begin{array}{l}\text { NOS/ } \\
\text { Jadad } \\
\text { score* }^{*}\end{array}$ \\
\hline $\begin{array}{l}\text { Lohsiriwat } \\
\text { et al. [34] }\end{array}$ & 2021 & $\begin{array}{l}\text { Prospective } \\
\text { cohort }\end{array}$ & $\begin{array}{l}\text { Colorectal cancer } \\
\text { resection }\end{array}$ & $\begin{array}{l}\text { Non-ERAS }(n=279) \\
\text { ERAS }(n=70)\end{array}$ & $\begin{array}{l}\text { Mean } 63.1 \\
\text { Mean } 62.1\end{array}$ & OS & 7 \\
\hline \multicolumn{8}{|c|}{ High adherence vs low adherence to ERAS } \\
\hline $\begin{array}{l}\text { Passeri et al. } \\
{[16]}\end{array}$ & 2020 & $\begin{array}{l}\text { Prospective } \\
\text { cohort }\end{array}$ & $\begin{array}{l}\text { Pancreatico- } \\
\text { duodenectomy }\end{array}$ & $\begin{array}{l}\text { High }(n=42) \\
\text { Low }(n=44)\end{array}$ & Median 67 & OS; DFS & 6 \\
\hline $\begin{array}{l}\text { Viannay et al. } \\
\text { [22] }\end{array}$ & 2019 & $\begin{array}{l}\text { Retrospective } \\
\text { cohort }\end{array}$ & Colectomy & $\begin{array}{l}\text { High }(n=52) \\
\text { Low }(n=154)\end{array}$ & $\begin{array}{l}\text { Mean } 68.2 \\
\text { Mean } 71.8\end{array}$ & OS; survival of metastasis & 7 \\
\hline $\begin{array}{l}\text { Gustafsson } \\
\text { et al. [23] }\end{array}$ & 2016 & $\begin{array}{l}\text { Retrospective } \\
\text { cohort }\end{array}$ & Colorectal resection & $\begin{array}{l}\text { High }(n=273) \\
\text { Low }(n=638)\end{array}$ & $\begin{array}{l}\text { Mean } 68.7 \\
\text { Mean } 69.6\end{array}$ & Local recurrence; OS; CSS & 7 \\
\hline $\begin{array}{l}\text { Pisarska et al. } \\
\text { [24] }\end{array}$ & 2019 & $\begin{array}{l}\text { Prospective } \\
\text { cohort }\end{array}$ & Colorectal resection & $\begin{array}{l}\text { High }(n=241) \\
\text { Low }(n=109)\end{array}$ & $\begin{array}{l}\text { Mean } 63.8 \\
\text { Mean } 64.9\end{array}$ & OS & 7 \\
\hline $\begin{array}{l}\text { St-Amour } \\
\text { et al. [25] }\end{array}$ & 2020 & $\begin{array}{l}\text { Retrospective } \\
\text { cohort }\end{array}$ & $\begin{array}{l}\text { Surgery for liver and } \\
\text { pancreatic cancer }\end{array}$ & $\begin{array}{l}\text { High }(n=42) \\
\text { Low }(n=91)\end{array}$ & Median 67 & $\begin{array}{l}\text { Days to RIOT in young patients; } \\
\text { DFS in young patients }\end{array}$ & 6 \\
\hline $\begin{array}{l}\text { Rubinkiewicz } \\
\text { et al. [26] }\end{array}$ & 2020 & $\begin{array}{l}\text { Retrospective } \\
\text { cohort }\end{array}$ & Gastrectomy & $\begin{array}{l}\text { High }(n=34) \\
\text { Low }(n=44)\end{array}$ & $\begin{array}{l}\text { Mean } 61.1 \\
\text { Mean } 61.3\end{array}$ & OS & 7 \\
\hline $\begin{array}{l}\text { Lohsiriwat } \\
\text { et al. [34] }\end{array}$ & 2021 & $\begin{array}{l}\text { Prospective } \\
\text { cohort }\end{array}$ & $\begin{array}{l}\text { Colorectal cancer } \\
\text { resection }\end{array}$ & $\begin{array}{l}\text { High }(n=232) \\
\text { Low }(n=88)\end{array}$ & $\begin{array}{l}\text { Mean } 62.5 \\
\text { Mean } 65.1\end{array}$ & OS & 7 \\
\hline \multicolumn{8}{|c|}{ Alteration of single item within ERAS protocol } \\
\hline $\begin{array}{l}\text { Curtis et al. } \\
{[27]}\end{array}$ & 2018 & $\begin{array}{l}\text { Prospective } \\
\text { cohort }\end{array}$ & Colorectal resection & $\begin{array}{l}\text { Laparoscopy surgery } \\
(n=383) \\
\text { Open surgery }(n= \\
373)\end{array}$ & Unclear & OS & 7 \\
\hline $\begin{array}{l}\text { Wang et al. } \\
{[29]}\end{array}$ & 2021 & $\begin{array}{l}\text { Retrospective } \\
\text { cohort }\end{array}$ & $\begin{array}{l}\text { Colorectal cancer } \\
\text { resection }\end{array}$ & $\begin{array}{l}\text { Laparoscopy }(\mathrm{n}= \\
125 \text { ) } \\
\text { Open surgery }(\mathrm{n}= \\
137 \text { ) }\end{array}$ & $\begin{array}{l}\text { Mean } 56.2 \\
\text { Mean } 57.1\end{array}$ & OS; DFS & 7 \\
\hline $\begin{array}{l}\text { Asklid et al. } \\
{[28]}\end{array}$ & 2017 & $\begin{array}{l}\text { Prospective } \\
\text { cohort }\end{array}$ & Colorectal resection & $\begin{array}{l}\text { Restrictive fluid } \\
\text { therapy }(n=145) \\
\text { Liberal fluid therapy } \\
(n=753)\end{array}$ & Mean 69.3 & Local recurrence; OD; CSD & 7 \\
\hline $\begin{array}{l}\text { Kato et al. } \\
{[35]}\end{array}$ & 2021 & $\begin{array}{l}\text { Retrospective } \\
\text { cohort }\end{array}$ & Rectosigmoid resection & $\begin{array}{l}\text { Early oral feeding ( } \mathrm{n} \\
=106) \\
\text { Conventional oral } \\
\text { feeding }(\mathrm{n}=95)\end{array}$ & $\begin{array}{l}\text { Median } 60 \\
\text { Median } 62\end{array}$ & Days to RIOT & 6 \\
\hline
\end{tabular}

RIOT return to intended oncologic therapy, OS overall survival, CSS cancer-specific survival, DFS disease-free survival, OD overall death, CSD cancer-specific death * Study evaluated by Jadad score

adherence to ERAS [23, 24, 34]. Two studies reported DFS [16, 25]; one showed that high adherence to ERAS could improve 3-year DFS [25], and the other did not find any difference [16]. One study showed that high adherence to ERAS could improve 5-year CSS [23], 2 studies reported that high adherence to ERAS had no effect on 3-year metastasis or 5-year recurrence [22, 23], and 1 study showed that high adherence to ERAS reduced the interval from surgery to RIOT [25].

\section{Alteration of one single item within ERAS protocol}

Four studies reported the effect of altering one single item within the ERAS protocol on long-term survival and local recurrence [27-29, 35]. The follow-up time ranged from 10 months to 5 years. Two studies compared laparoscopic with open surgery within the ERAS protocol, of which, 1 study revealed improvements in OS with laparoscopic surgery [27], and the other did not find any difference [29]. One study compared restrictive with liberal fluid therapy within the ERAS protocol, and revealed improvements in OS with restrictive fluid therapy, but no differences were found in local recurrence [28]. One study compared early oral feeding with conventional oral feeding within ERAS protocol, and found no difference in days to RIOT after surgery [35].

\section{Discussion}

Since the concept of ERAS was proposed more than 20 years ago, these regimen has been widely applied in cardiac surgery, general surgery, neurosurgery, head and neck surgery, thoracic surgery, gynecologic surgery, urinary surgery, and orthopedic surgery. Clinical evidence 
Table 2 ERAS protocol elements in the included studies

\begin{tabular}{|c|c|c|c|c|c|c|c|c|c|c|c|c|c|c|c|c|c|c|c|c|c|c|c|c|c|c|}
\hline References No. & 10 & 11 & 12 & 13 & 14 & 15 & 16 & 17 & 18 & 19 & 20 & 21 & 22 & 23 & 24 & 25 & 26 & 27 & 28 & 29 & 30 & 31 & 32 & 33 & 34 & 35 \\
\hline Education and counseling & - & + & - & + & + & + & + & + & + & - & + & + & + & + & + & + & + & + & + & + & + & + & + & + & + & - \\
\hline Bowel preparation & - & + & + & + & - & + & + & + & - & + & - & + & + & + & + & + & - & + & + & + & + & - & - & - & + & - \\
\hline Carbohydrate loading & - & + & + & + & + & + & + & + & - & + & + & + & + & + & + & + & + & + & + & - & + & - & - & - & + & + \\
\hline Regional anesthesia & + & + & - & + & + & + & + & + & + & - & + & + & - & + & 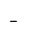 & - & + & + & + & + & - & + & + & + & - & - \\
\hline Steroids & - & - & - & - & - & - & - & + & - & - & + & - & + & - & - & 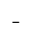 & - & - & - & - & + & - & - & - & - & \\
\hline Body ter & - & + & - & + & + & + & + & - & + & - & - & + & + & + & - & - & - & - & + & + & + & - & + & + & + & - \\
\hline Fluid therapy & - & + & - & + & + & + & + & + & - & + & + & - & + & - & + & - & + & + & - & + & + & + & + & + & + & \\
\hline Micro-invasive surgery & - & + & - & - & + & + & + & - & - & - & - & - & + & - & + & - & + & + & - & + & + & + & - & - & + & \\
\hline Multimodal analgesia & + & + & + & + & + & + & + & + & + & + & + & + & + & - & + & + & + & + & - & + & + & + & + & + & + & \\
\hline NG tube & + & + & + & + & + & - & + & + & + & - & - & + & + & - & + & - & + & + & - & + & + & - & + & + & + & - \\
\hline Drainage & + & + & + & - & + & - & + & - & + & - & - & + & + & - & + & + & + & + & - & + & + & - & + & + & + & \\
\hline Urinary catheter & - & + & - & + & + & - & + & - & + & - & - & - & + & - & + & - & + & - & - & + & - & + & + & + & + & - \\
\hline Prophylactic antithrombosis & - & + & - & - & + & + & + & - & - & - & - & - & + & - & + & + & $T$ & - & - & - & ${ }_{T}$ & - & + & + & + & \\
\hline Prophylactic antibiotics & - & + & - & - & - & - & + & - & - & - & + & + & - & - & + & + & + & - & - & - & + & - & + & - & + & + \\
\hline Prophylactic anti-emetics & - & + & - & - & - & + & + & - & + & - & - & - & + & - & + & - & - & - & - & - & + & - & - & - & + & - \\
\hline Early oral feeding & + & + & - & + & + & + & + & + & + & + & + & + & + & - & + & - & + & + & + & + & - & + & + & + & + & + \\
\hline Early mobilization & + & + & + & + & + & + & + & + & + & + & + & + & + & + & + & - & + & + & + & + & + & + & + & + & + & - \\
\hline Discharge planning & - & - & - & + & - & - & - & + & + & - & - & - & - & - & - & - & - & - & - & - & - & - & - & - & - & - \\
\hline Others & - & + & - & - & - & - & + & - & - & - & - & - & + & + & - & + & - & - & + & + & + & - & + & + & + & + \\
\hline Elements ( $n$ ) & 6 & 17 & 6 & 12 & 13 & 12 & 17 & 11 & 11 & 6 & 9 & 11 & 16 & 7 & 14 & 8 & 13 & 11 & 8 & 13 & 15 & 8 & 13 & 12 & 16 & 3 \\
\hline
\end{tabular}

"+", element explicitly listed in ERAS protocol; "-", element not explicitly listed in ERAS protocol

has proved that ERAS can improve short-term postoperative outcomes in both cancer and non-cancer surgeries. For cancer patients, quality of life and long-term survival are the most important factor. However, whether the short-term benefits of ERAS are associated with longterm benefits in patients undergoing cancer surgeries has not yet been verified.

The results of this review showed that ERAS improved the on-time initiation and completion of RIOT after cancer surgeries. Various factors are considered when deciding to start adjuvant chemotherapy after surgery, of which, the patient's condition is the most important one $[36,37]$. ERAS improves short-term outcomes including patient conditions, so cancer patients undergoing ERAS can receive on-time and higher-rate chemotherapy after surgery. The interval from surgery to adjuvant chemotherapy is commonly 30 to 60 days; the interval in the included studies ranged from 33 days to 68 days, so the initiation or completion of RIOT is a mid-term outcome measure [38], not a short-term or long-term measure. This review showed that ERAS could not improve longterm OS or CSS in the majority of the included studies. Theoretically, improved short-term outcomes are postulated to be associated with long-term outcomes; however, the results of this review suggested that ERASinduced improvement of mid-term oncologic outcomes was not associated with long-term survival, and no studies have compared the effects of ERAS and nonERAS on local recurrence and metastasis until now. It was reported that the long-term prognosis after bladder cancer surgery was determined by tumor stage, presence of metastasis at surgery, and resection status [12]. ERAS might not play the pivotal role in long-term prognosis.

Adherence to ERAS is critical for short-term outcomes. Higher adherence is associated with better shortterm outcomes $[39,40]$. This review included 6 studies comparing long-term survival between high adherence to REAS and low adherence to ERAS [16, 22-24, 34], and half reported improvements in survival by high adherence, and half did not. One study reported improvements in on-time initiation of RIOT with high adherence [25]. Till now, the criteria of the adherence to ERAS have not been well defined, and the level of high adherence ranged from 67 to $85 \%$ in the included 7 studies. Higher adherence is more difficult to implement, and $70 \%$ adherence to ERAS is considered a common standard of high adherence and an achievable target in the clinic [40].

Within the ERAS protocol, restrictive fluid therapy could reduce 5-year OD and CSD in one study, while the effects on long-term survival were controversial between laparoscopic and open surgery in two studies, days to RIOT was not improved by early oral feeding compared with conventional oral feeding. Till now, 
Table 3 Effects of ERAS on oncologic and long-term outcomes

\begin{tabular}{|c|c|c|c|c|c|c|}
\hline \multicolumn{3}{|l|}{ Outcomes } & Study & $P$ value & Findings & $\begin{array}{l}\text { Follow-up } \\
\text { time }\end{array}$ \\
\hline \multirow[t]{31}{*}{ Non-ERAS vs ERAS } & \multirow{12}{*}{\multicolumn{2}{|c|}{ OS }} & Pang et al. [11] & $P=0.9$ & NS & 10 years \\
\hline & & & $\begin{array}{l}\text { Ziegelmueller } \\
\text { et al. [12] }\end{array}$ & $P=0.550$ & NS & 7 years \\
\hline & & & Oakley et al. [10] & $P=0.57$ & NS & 5 years \\
\hline & & & Yang et al. [14] & $P=0.007$ & ERAS improved OS & 5 years \\
\hline & & & Ye et al. [13] & $P=0.066$ & NS & 3 years \\
\hline & & & Passeri et al. [16] & $P=0.72$ & NS & 2 years \\
\hline & & & Wang et al. [29] & / & NS & $\begin{array}{l}10-20 \\
\text { months }\end{array}$ \\
\hline & & & Zhang et al. [30] & $P=0.035$ & ERAS improved OS & 3 years \\
\hline & & & Quiram et al. [31] & $P=0.464$ & NS & 5 years \\
\hline & & & Zhu et al. [32] & $P=0.810$ & NS & 1 year \\
\hline & & & Tian et al. [33] & $P=0.013$ & ERAS improved OS & 5 years \\
\hline & & & $\begin{array}{l}\text { Lohsiriwat et al. } \\
\text { [34] }\end{array}$ & $P=0.014$ & ERAS improved OS & 5 years \\
\hline & \multirow{3}{*}{\multicolumn{2}{|c|}{ CSS }} & Pang et al. [11] & $P=0.9$ & NS & 10 years \\
\hline & & & $\begin{array}{l}\text { Ziegelmueller } \\
\text { et al. [12] }\end{array}$ & $P=0.725$ & NS & 7 years \\
\hline & & & Tian et al. [33] & $P=0.033$ & ERAS improved CSS & 5 years \\
\hline & \multirow{4}{*}{\multicolumn{2}{|c|}{ DFS }} & Passeri et al. [16] & $P=0.38$ & NS & 2 years \\
\hline & & & Wang et al. [29] & / & NS & $\begin{array}{l}10-20 \\
\text { months }\end{array}$ \\
\hline & & & Zhang et al. [30] & $P=0.007$ & ERAS improved DFS & 3 years \\
\hline & & & Quiram et al. [31] & $P=0.272$ & NS & 5 years \\
\hline & \multicolumn{2}{|c|}{ Recurrence } & Quiram et al. [31] & $P=0.157$ & NS & 3 years \\
\hline & \multicolumn{2}{|c|}{ Metastasis } & Quiram et al. [31] & $P=0.129$ & NS & 5 years \\
\hline & \multirow[t]{3}{*}{$\begin{array}{l}\text { Life } \\
\text { quality }\end{array}$} & $\begin{array}{l}\text { Physical } \\
\text { functioning }\end{array}$ & Liu et al. [15] & $P=0.038$ & ERAS improved life quality & 6 months \\
\hline & & Nausea/vomiting & & $P=0.048$ & & \\
\hline & & Motor dysfunction & & $P=0.019$ & & \\
\hline & \multirow[t]{7}{*}{ RIOT } & Failure to RIOT & Day et al. [17] & $P=0.373$ & NS & / \\
\hline & & Days to RIOT & Day et al. [17] & $P=0.134$ & NS & \\
\hline & & & Lohsiriwat [18] & $P=0.009$ & ERAS reduced interval to RIOT & \\
\hline & & & Nelson et al. [20] & $P=0.364$ & NS & \\
\hline & & & Li et al. [21] & $P=0.000$ & ERAS reduced interval to RIOT & \\
\hline & & $\begin{array}{l}\text { Rate of on time to } \\
\text { RIOT }\end{array}$ & $\begin{array}{l}\text { Hassinger et al. } \\
\text { [19] }\end{array}$ & $P=0.022$ & $\begin{array}{l}\text { ERAS improved the on-time initi- } \\
\text { ation of RIOT }\end{array}$ & \\
\hline & & $\begin{array}{l}\text { Rate of completing } \\
\text { RIOT }\end{array}$ & Nelson et al. [20] & $P<0.001$ & ERAS improved RIOT completion & \\
\hline \multirow[t]{5}{*}{ High adherence vs low adherence } & \multirow{5}{*}{\multicolumn{2}{|c|}{ OS }} & $\begin{array}{l}\text { Gustafsson et al. } \\
\text { [23] }\end{array}$ & $P<0.001$ & High adherence improved OS & 5 years \\
\hline & & & $\begin{array}{l}\text { Lohsiriwat et al. } \\
\text { [34] }\end{array}$ & $P=0.007$ & High adherence improved OS & 5 years \\
\hline & & & Viannay et al. [22] & $P=0.632$ & NS & 3 years \\
\hline & & & Pisarska et al. [24] & $\begin{array}{l}P= \\
0.0007\end{array}$ & High adherence improved OS & \\
\hline & & & $\begin{array}{l}\text { Rubinkiewicz et al. } \\
{[26]}\end{array}$ & $P=0.75$ & NS & \\
\hline
\end{tabular}


Table 3 Effects of ERAS on oncologic and long-term outcomes (Continued)

\begin{tabular}{|c|c|c|c|c|c|}
\hline \multicolumn{2}{|l|}{ Outcomes } & \multirow{2}{*}{$\begin{array}{l}\text { Study } \\
\text { Passeri et al. [16] }\end{array}$} & \multirow{2}{*}{$\begin{array}{l}P \text { value } \\
P=0.14\end{array}$} & \multirow{2}{*}{$\begin{array}{l}\text { Findings } \\
\text { NS }\end{array}$} & \multirow{2}{*}{$\begin{array}{l}\text { Follow-up } \\
\text { time }\end{array}$} \\
\hline & & & & & \\
\hline & CSS & $\begin{array}{l}\text { Gustafsson et al. } \\
\text { [23] }\end{array}$ & $P=0.020$ & High adherence improved CSS & 5 years \\
\hline & DFS & $\begin{array}{l}\text { St-Amour et al. } \\
\text { [25] }\end{array}$ & $P=0.000$ & High adherence improved DFS & 3 years \\
\hline & & Passeri et al. [16] & $P=0.81$ & NS & 2 years \\
\hline & Survival of metastasis & Viannay et al. [22] & $P=0.668$ & NS & 3 years \\
\hline & Local recurrence & $\begin{array}{l}\text { Gustafsson et al. } \\
{[23]}\end{array}$ & $P=0.211$ & NS & 5 years \\
\hline & $\begin{array}{l}\text { Days to RIOT in young } \\
\text { patients }\end{array}$ & $\begin{array}{l}\text { St-Amour et al. } \\
{[25]}\end{array}$ & $P=0.001$ & $\begin{array}{l}\text { High adherence reduced interval } \\
\text { to RIOT }\end{array}$ & 3 years \\
\hline \multirow{7}{*}{$\begin{array}{l}\text { Alteration of single item within } \\
\text { ERAS protocol }\end{array}$} & OS & Curtis et al. [27] & $P=0.009$ & Laparoscopy surgery improved OS & 5 years \\
\hline & & Wang et al. [29] & / & NS & $\begin{array}{l}10-20 \\
\text { months }\end{array}$ \\
\hline & DFS & Wang et al. [29] & / & NS & $\begin{array}{l}10-20 \\
\text { months }\end{array}$ \\
\hline & Local recurrence & Asklid et al. [28] & $P=0.981$ & NS & 5 years \\
\hline & $\mathrm{OD}$ & & $P=0.006$ & \multirow{2}{*}{$\begin{array}{l}\text { Restrictive fluid therapy improved } \\
\text { survival }\end{array}$} & \\
\hline & CSD & & $P=0.008$ & & \\
\hline & Days to RIOT & Kato et al. [35] & $P=0.08$ & NS & / \\
\hline
\end{tabular}

OS overall survival, CSS cancer-specific survival, DFS disease-free survival, RIOT return to intended oncologic therapy, OD overall death, CSD cancer-specific death, NS no significance

studies on alteration of one single item within ERAS protocol were scarce. Furthermore, the ERAS protocol is a combination therapy, and the pre-, intra-, and postoperative items are combined together to improve postoperative outcomes. So alteration of one single item within ERAS protocol might not be so important for the oncologic and long-term outcomes. In the 26 included studies in this review, the most frequently used components of ERAS included patient education and counseling, avoidance of bowel preparation, carbohydrate loading, regional anesthesia, targeted fluid therapy, multimodal analgesia, early removal of nasogastric tubes and drainage, early oral feeding, and mobilization. However, the number of ERAS components ranged from 3 to 18 in these studies, and different numbers of ERAS components might cause different outcomes. Perioperative steroids or discharge planning were used in few studies. Therefore, the standard ERAS protocol should be used for each type of cancer surgery in accordance with the ERAS protocols in the ERAS interactive audit system, so that the oncologic and long-term outcomes under a defined framework can be fully assessed.

There are some limitations in this systematic review. First, tumor entities and surgical procedures were quite different; although in each study the results were comparable between groups, the results between studies were not comparable. Second, ERAS protocols and the follow-up time in these included trials were not uniform, so that meta-analysis could not be conducted. Third, DFS is a most important endpoint in oncologic studies, but only few studies reported the effect of ERAS on long-term DFS; regardless of the fact that ERAS could improve RIOT after surgery, the long-term oncologic outcomes after ERAS were still unclear. Fourth, the majority of the included studies were of cohort studies, and large RCTs are needed to verify the effects of the ERAS protocol on oncologic and long-term outcomes in the future.

\section{Conclusions}

This systematic review identified 26 studies with variable patient populations, cancer surgeries, and ERAS protocol implementation. Our results showed that ERAS protocols in cancer surgeries can improve the on-time initiation and completion of adjuvant chemotherapy after surgery, and high adherence to ERAS lead to better outcomes than low adherence. Based on the current evidence, it is difficult to determine whether the ERAS protocol is associated with recurrence or metastasis and long-term survival. Future efforts should be directed towards the application of a standard ERAS protocol in each type of cancer surgery and evaluation of its impact using larger, comparative multi-center studies at highand low-volume medical centers. 


\section{Abbreviations}

ERAS: Enhanced recovery after surgery; OS: Overall survival; CSS: Cancerspecific survival; DFS: Disease-free survival; RIOT: Return to intended oncologic therapy

\section{Supplementary Information}

The online version contains supplementary material available at https://doi. org/10.1186/s12957-021-02306-2.

Additional file 1. PRISMA 2009 Checklist.

Additional file 2. Search strategies for other database.

\section{Acknowledgements}

The authors declare that no acknowledgements have to be made.

\section{Authors' contributions}

$\mathrm{HL}$ participated in the design of the review and revised the manuscript. QP collected the data and drafted the manuscript. LD and YJ collected the data and proofread the manuscript. The authors read and approved the final version.

\section{Funding}

Not applicable.

\section{Availability of data and materials}

The data-sets supporting the conclusion are included in the article.

\section{Declarations}

Ethics approval and consent to participate

Not applicable.

\section{Consent for publication}

Not applicable.

\section{Competing interests}

The authors declare that they have no competing interests.

Received: 3 March 2021 Accepted: 17 June 2021

\section{Published online: 29 June 2021}

\section{References}

1. Kehlet H. Multimodal approach to control postoperative pathophysiology and rehabilitation. Br J Anaesth. 1997;78(5):606-17. https://doi.org/10.1093/ $\mathrm{bja} / 78.5 .606$

2. Tejedor P, Pastor C, Gonzalez-Ayora S, Ortega-Lopez M, Guadalajara H, Garcia-Olmo D. Short-term outcomes and benefits of ERAS program in elderly patients undergoing colorectal surgery: a case-matched study compared to conventional care. Int J Color Dis. 2018;33(9):1251-8. https:// doi.org/10.1007/s00384-018-3057-z.

3. Oh C, Moriarty J, Borah BJ, Mara KC, Harmsen WS, Saint-Cyr M, et al. Cost analysis of enhanced recovery after surgery in microvascular breast reconstruction. J Plast Reconstr Aesthet Surg. 2018;71(6):819-26. https://doi. org/10.1016/.j.jps.2018.02.018.

4. Ljungqvist O, Scott M, Fearon KC. Enhanced recovery after surgery: a review. JAMA Surg. 2017;152(3):292-8. https://doi.org/10.1001/jamasurg.2016.4952.

5. Jing $X$, Zhang $B$, Xing $S$, Tian $L$, Wang $X$, Zhou $M$, et al. Cost-benefit analysis of enhanced recovery after hepatectomy in Chinese Han population. Medicine. 2018;97:e1 1957. https://doi.org/10.1097/MD.0000000000011957.

6. Brown JK, Singh K, Dumitru R, Chan E, Kim MP. The benefits of enhanced recovery after surgery programs and their application in cardiothoracic surgery. Methodist Debakey Cardiovasc J. 2018;14:77-88. https://doi.org/1 0.14797/mdcj-14-2-77.

7. Biagi JJ, Raphael MJ, Mackillop WJ, Kong W, King WD, Booth CM. Association between time to initiation of adjuvant chemotherapy and survival in colorectal cancer: a systematic review and meta-analysis. JAMA. 2011;305(22):2335-42. https://doi.org/10.1001/jama.2011.749.

8. Khuri SF, Henderson WG, DePalma RG, Mosca C, Healey NA, Kumbhani DJ, et al. Determinants of long-term survival after major surgery and the adverse effect of postoperative complications. Ann Surg. 2005;242(3):32641. https://doi.org/10.1097/01.sla.0000179621.33268.83.

9. Moher D, Liberati A, Tetzlaff J, Altman DG, PRISMA Group. PRISMA Group, Preferred reporting items for systematic reviews and meta-analysis: the PRIS MA statement. J Clin Epidemiol. 2009;62(7):1006-12. https://doi.org/10.1371/ journal.pmed.1000097.

10. Oakley B, Lamb C, Vohra R, Catton J. Achieving long term survival in oesophagectomy patients aged over 75. Ann med surg. 2016;9:15-21. https://doi.org/10.1016/j.amsu.2016.05.016.

11. Pang KH, Groves R, Venugopal S, Noon AP, Catto JWF. Prospective implementation of enhanced recovery after surgery protocols to radical cystectomy. Eur Urol. 2018;73(3):363-71. https://doi.org/10.1016/j.eururo.201 7.07.031.

12. Ziegelmueller BK, Jokisch JF, Buchner A, Grimm T, Kretschmer A, Schulz GB, et al. Long-term follow-up and oncological outcome of patients undergoig radical cystectomy for bladder cacner following an enhanced recovery after surgery protocol: results of a large randomized, prospective, single-center study. Urol Int. 2020;104(1-2):55-61. https://doi.org/10.1159/000504236.

13. Ye S. Effect of ERAS on short-term and long-term efficacy of surgical treatment for young and middle-aged patients with gastric cancer. Pract J Cancer (Chinese). 2017:32:1511-4.

14. Yang FZ, Wang H, Wang DS, Niu ZJ, Li SK, Zhang J, et al. The effect of perioperative ERAS pathway management on short- and long-term outcomes of gastric cancer patients. Zhonghua Yi Xue Za Zhi. 2020;100(12): 922-7. https://doi.org/10.3760/cma.j.cn112137-20190711-01325.

15. Liu B, Liu S, Wang Y, Lu D, Chen L, Zheng T, et al. Impact of neurosurgical enhanced recovery after surgery (ERAS) program on health-related quality of life in glioma patients: a secondary analysis of a randomized controlled trial. J Neuro-Oncol. 2020;148(3):555-67. https://doi.org/10.1007/s1 1060-02003548-y.

16. Passeri M, Lyman WB, Murphy K, lannitti D, Martinie J, Baker E, et al. Implementing an ERAS protocol for pancreaticoduodenectomy does not affect oncologic outcomes. Am Surg. 2020;86:e81-3 32106919.

17. Day RW, Cleeland CS, Wang XS, Fielder S, Calhoun J, Conrad C, et al. Patient-reported outcomes accurately measure the value of an enhanced recovery program in liver surgery. J Am Coll Surg. 2015;221(6):1023-30. https://doi.org/10.1016/j.jamcollsurg.2015.09.011.

18. Lohsiriwat V. Enhanced recovery after surgery vs conventional care in emergency colorectal surgery. World J Gastroenterol. 2014;20(38):13950-5. https://doi.org/10.3748/wjg.v20.i38.13950.

19. Hassinger TE, Mehaffey JH, Martin AN, Bauer-Nilsen K, Turrentine FE, Thiele $\mathrm{RH}$, et al. Implementation of an enhanced recovery protocol is associated with on-time initiation of adjuvant chemotherapy in colorectal cancer. Dis Colon Rectum. 2019;62(11):1305-15. https://doi.org/10.1097/DCR. 0000000000001486

20. Nelson DB, Mehran RJ, Mitchell KG, Correa AM, Sepesi B, Antonoff MB, et al. Enhanced recovery after thoracic surgery is associated with improved adjuvant chemotherapy completion for non-small cell lung cancer. J Thorac Cardiovasc Surg. 2019;158(1):279-86. https://doi.org/10.1016/j.jtcvs.2 019.03.009.

21. Li M, Wang X, Shen R, Wang S, Zhu D. Advancing the time to the initiation of adjuvant chemotherapy and improving postoperative outcome: enhanced recovery after surgery in pancreaticoduodenectomy. Am Surg. 2020;86(4):293-9. 32391752. https://doi.org/10.1177/000313482008600424.

22. Viannay P, Hamy A, Jaouen R, Caroli-Bosc FX, Luel C, Vasseur S, et al. Does enhanced recovery improve the survival rates of patients 3 years after undergoing surgery to remove a tumor in the colon? Int J Color Dis. 2019; 34(3):441-9. https://doi.org/10.1007/s00384-018-3205-5.

23. Gustafsson UO, Oppelstrup H, Thorell A, Nygren J, Ljungqvist O. Adherence to the ERAS protocol is associated with 5-Year survival after colorectal cancer surgery: a retrospective Cohort study. World J Surg. 2016;40(7):17417. https://doi.org/10.1007/s00268-016-3460-y.

24. Pisarska M, Torbicz G, Gajewska N, Rubinkiewicz M, Wierdak M, Major P, et al. Compliance with the ERAS protocol and 3-year survival after laparoscopic surgery for non-metastatic colorectal cancer. World J Surg. 2019:43(10):2552-60. https://doi.org/10.1007/s00268-019-05073-0.

25. St-Amour P, St-Amour P, Joliat GR, Eckert A, Labgaa I, Roulin D, et al. Impact of ERAS compliance on the delay between surgery and adjuvant chemotherapy in hepatobiliary and pancreatic malignancies. Langenbeck's Arch Surg. 2020;405(7):959-66. https://doi.org/10.1007/ s00423-020-01981-1. 
26. Rubinkiewicz M, Pisarska M, Zarzycki P, Truszkiewicz K, Witowaki J, Su M, et al. High compliance to ERAS protocol does not improve overall survival in patients treated for resectable advanced gastric cancer. Videosurgery Miniinv. 2020;15(4):553-9. https://doi.org/10.5114/wiitm.2020.92833.

27. Curtis NJ, Taylor M, Fraser L, Salib E, Noble E, Hipkiss R, et al. Can the combination of laparoscopy and enhanced recovery improve long-term survival after elective colorectal cancer surgery? Int J Color Dis. 2018;33(2): 231-4. https://doi.org/10.1007/s00384-017-2935-0.

28. Asklid D, Segelman J, Gedda C, Hjern F, Pekkari K, Gustafsson UO. The impact of perioperative fluid therapy on short-term outcomes and 5-year survival among patients undergoing colorectal cancer surgery - a prospective cohort study within an ERAS protocol. EJSO. 2017;43(8):1433-9 https://doi.org/10.1016/j.ejso.2017.04.003.

29. Wang B, Wu Z, Zhang R, Chen Y, Dong J, Qi X. Retrospective analysis of safety and efficacy of enhanced recovery pathways in stage II-III colorectal cancer patients submitted to surgery and adjuvant therapy. World J Surg Oncol. 2021;19(1):99. https://doi.org/10.1186/s12957-021-02203-8.

30. Zhang Z, Gu W, Zhang Y. Impact of enhanced recovery after surgery on long-term outcomes and postoperative recovery in patients undergoing hepatectomy: a retrospective cohort study. Cancer Manag Res. 2021;13: 2681-90. https://doi.org/10.2147/CMAR.S301859.

31. Quiram BJ, Crippa J, Grass F, Lovely JK, Behm KT, Colibaseanu DT, et al. Impact of enhanced recovery on oncological outcomes following minimally invasive surgery for rectal cancer. Br J Surg. 2019;106(7):922-9. https://doi. org/10.1002/bjs.11131.

32. Zhu J, Li X, Li H, Liu Z, Ma J, Kou J, et al. Enhanced recovery after surgery pathways benefit patients with soft pancreatic texture following pancreaticoduodenectomy. Am J Surg. 2020;219(6):1019-23. https://doi. org/10.1016/j.amjsurg.2019.08.002.

33. Tian YL, Cao SG, Liu XD, Li ZQ, Liu G, Zhang XQ, et al. Short- and long-term outcomes associated with enhanced recovery after surgery protocol vs conventional management in patients undergoing laparoscopic gastrectomy. World J Gastroenterol. 2020;26(37):5646-60. https://doi.org/1 0.3748/wjg.v26.i37.5646.

34. Lohsiriwat V, Lertbannaphong S, Polakla B, Riansuwan W. Implementation of enhanced recovery after surgery and its increasing compliance improved 5year overall survival in resectable stage III colorectal cancer. Updat Surg. 2021. https://doi.org/10.1007/s13304-021-01004-8 Epub ahead of print.

35. Kato K, Omatsu K, Okamoto S, Matoda M, Nomura H, Tanigawa T, et al. Early oral feeding is safe and useful after rectosigmoid resection with anastomosis during cytoreductive surgery for primary ovarian cancer. World J Surg Oncol. 2021;19(1):77. https://doi.org/10.1186/s12957-021-02186-6.

36. Kutner JS, Vu KO, Prindiville SA, Byers TE. Patient age and cancer treatment decisions. Patient and physician views. Cancer Pract. 2000;8(3):114-9. https:// doi.org/10.1046/j.1523-5394.2000.83004.x.

37. Merkow RP, Bentrem DJ, Mulcahy MF, Chung JW, Abbott DE, Kmiecik TE, et al. Effect of postoperative complications on adjuvant chemotherapy use for stage III colon cancer. Ann Surg. 2013;258(6):847-53. https://doi.org/10.1 097/SLA.0000000000000312.

38. Strouch MJ, Zhou G, Fleshman JW, Birnbaum EH, Hunt SR, Mutch MG. Time to initiation of postoperative chemotherapy: an outcome measure for patients undergoing laparoscopic resection for rectal cancer. Dis Colon Rectum. 2013;56(8):945-51. https://doi.org/10.1097/DCR.0b013e318290ce30.

39. Group EC. The impact of enhanced recovery protocol compliance on elective colorectal cancer resection: results from an international registry. Ann Surg. 2015;261(6):1153-9. https://doi.org/10.1097/SLA.0000000000001029.

40. Gustafsson UO, Hausel J, Thorell A, Ljungqvist O, Soop M, Nygren J, et al. Adherence to the enhanced recovery after surgery protocol and outcome after colorectal cancer surgery. Arch Surg. 2011;146(5):571-7. https://doi. org/10.1001/archsurg.2010.309.

\section{Publisher's Note}

Springer Nature remains neutral with regard to jurisdictional claims in published maps and institutional affiliations.

Ready to submit your research? Choose BMC and benefit from:

- fast, convenient online submission

- thorough peer review by experienced researchers in your field

- rapid publication on acceptance

- support for research data, including large and complex data types

- gold Open Access which fosters wider collaboration and increased citations

- maximum visibility for your research: over $100 \mathrm{M}$ website views per year

At $\mathrm{BMC}$, research is always in progress.

Learn more biomedcentral.com/submissions 